\title{
In search of the shortest regimen: fractionation of a fully isoeffective combination of hyperfractionated and hypofractionated treatment
}

\author{
Andrej Strojnik \\ Unit of Radiophysics, Department of Radiotherapy, Institute of Oncology, Ljubljana, Slovenia
}

Purpose. To analyze the possibility of reducing the number of fractions but maintaining the full biological effect of radiotherapy by varying the dose per fraction.

Methods. An arbitrary treatment with a constant dose per fraction is substituted for a fully isoeffective combination of a hyperfractionated and hypofractionated treatment. The number of fractions of the combined treatment is derived. All calculations are based on the linear-quadratic model.

Conclusions. Standard uniform fractionation requires the fewest fractions. Any variation in dose per fraction increases the number of fractions of a fully isoeffective treatment.

Key words: radiotherapy; fractionation; LQ model

\section{Introduction}

The primary goal of radiotherapy is to deliver sufficient radiation dose to destroy the clonogenic cancer cells while causing as little damage as possible to the normal cells. A contribution towards this objective is made by dividing the total therapeutic dose into fractions allowing radiation damage repair and recovery in healthy tissues and possibly reoxygenation and reassortment in tumors between consecutive treatment sessions. ${ }^{1-3}$ The fractionation prolongs the therapy making it also a logistic issue. Most treatment

Received 24 June 2008

Accepted 15 July 2008

Correspondence to: Andrej Strojnik, Bsc Phys, Unit of Radiophysics, Department of Radiotherapy, Institute of Oncology Ljubljana, Zaloska cesta 2, SI - 1000 Ljubljana, Slovenia; Phone: +386 15879 631; Fax: +386 15879 400; E-mail: astrojnik@onko-i.si regimens prescribe a constant dose per fraction throughout the treatment. In this article we look at the possibility of reducing the number of treatment fractions by replacing the constant dose per fraction schedule with an isoeffective combination of hyperfractionated and hypofractionated treatments.

\section{Methods and results}

Using the linear-quadratic (LQ) model Joiner illustrated how an incorrect dose delivered in the first few fractions can be counterbalanced by modifying the dose per fraction in the rest of the treatment to achieve the same biological effect as with the intended fractionation. ${ }^{4}$ In a few examples he calculated that the cumulative number of fractions exceeded that of the initially prescribed treatment. Bortfeld and 
Paganetti suspected this to always be the case $^{5}$, but did not prove it. In this paper we analyze the number of fractions in an isoeffective treatment where we deliberately deliver different doses per fraction without any other changes to the treatment plan, i.e. we vary the absolute dose but preserve the relative dose distribution.

Joiner derived his equations assuming all the tissues received equal absorbed dose, i.e. they all lay on the $100 \%$ isodose. We start by generalizing the validity of these equations to all isodoses. Let $d$ be the dose per fraction and let $D$ be the total dose in a treatment that consists of $n=D / d$ fractions. Also let the subscripts $H$ and $h$ denote the same quantities belonging to the hyperfractionated and hypofractionated part of the isoeffective treatment respectively, so that $d_{H}<d<d_{h}$. Further let $k$ be the relative isodose of interest. Now we request the biologically effective dose $(B E D)$ of the constant dose per fraction treatment be equal to that of the mixed regime

$B E D=B E D_{H}+B E D_{h}$

In the LQ model this translates to (after multiplying with $\alpha / \beta$ and relating to the $k$ isodose)

$k D(\alpha / \beta+k d)=k D_{H}\left(\alpha / \beta+k d_{H}\right)+k D_{h}\left(\alpha / \beta+k d_{h}\right)$

Dividing the equation by $k \neq 0$ and separating the terms with $\alpha / \beta$ and $k$ we get

$\alpha / \beta\left(D-D_{H}-D_{h}\right)=k\left(D_{H} d_{H}+D_{h} d_{h}-D d\right)$

For the equation to be valid for all the values of $\alpha / \beta$ and $k$, the following has to hold

$D_{H}+D_{h}=D$

$D_{H} d_{H}+D_{h} d_{h}=D d$

The above equations are the same as derived by Joiner, but now enjoy broader validity.

Next we look at the number of fractions in such a treatment

$n_{H}+n_{h}=\left(D_{H} d_{h}+D_{h} d_{H}\right) / d_{H} d_{h}$

The expression on the right calls for some maneuvering and application of equations [1] and [2]

$n_{H}+n_{h}=\left[\left(D_{H}+D_{h}\right)\left(d_{H}+d_{h}\right)-D_{H} d_{H}-D_{h} d_{h}\right] / d_{H} d_{h}=D\left(d_{H}+d_{h}-d\right) / d_{H} d_{h}$

after which we arrive to

$n_{H}+n_{h}=n\left[1+\left(d_{h}-d\right)\left(d-d_{H}\right) / d_{H} d_{h}\right]$ 


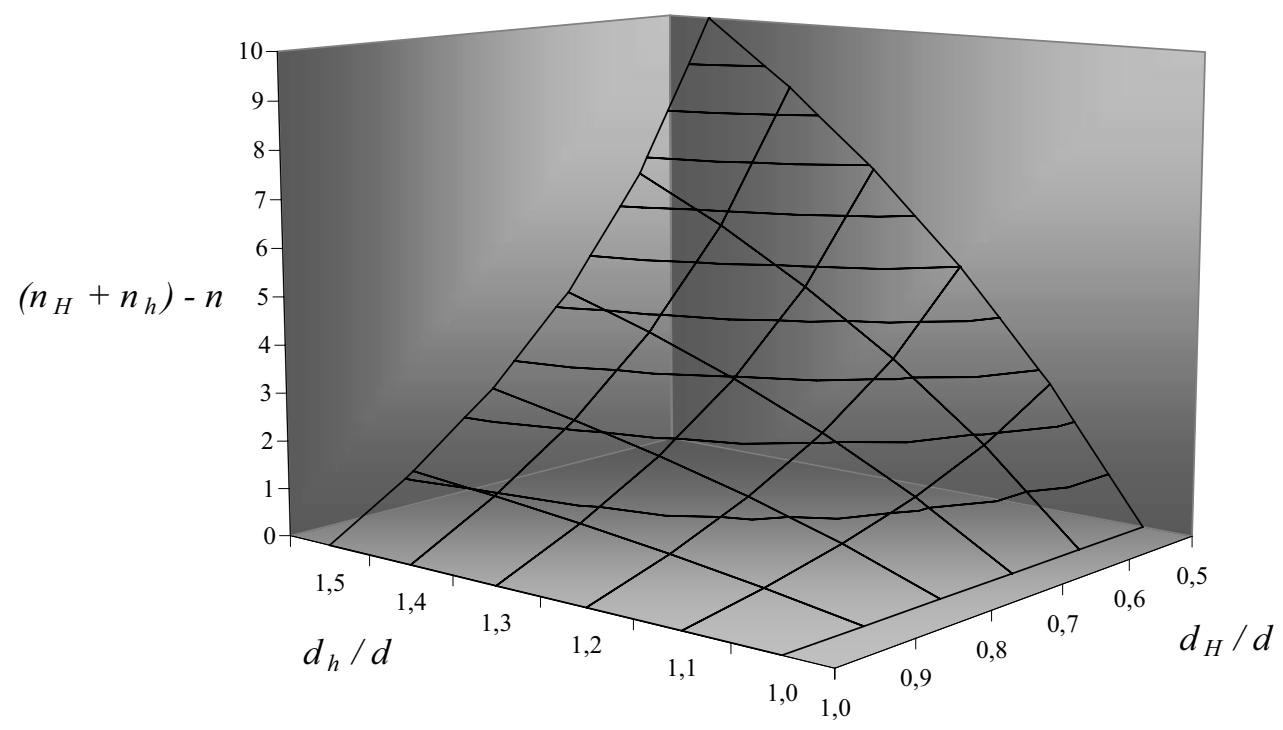

Figure 1. The chart shows the increase in number of fractions when moving from the standard to the combined regimen as a continuous function of relative doses per fraction $d_{H} / d$ and $d_{h} / d$. The number of fractions in the standard regimen $n$ is in this example assumed to be 30 .

Reminding ourselves that $d_{H}<d<d_{h}$, we clearly observe that the value in the square brackets is greater than 1 . This brings us to the conclusion

$$
n_{H}+n_{h}>n
$$

An example of this inequality is given in Figure 1.

\section{Conclusions}

Not only is a treatment with a constant dose per fraction the most practical and the easiest to manage among all the isoeffective schedules, it also involves the fewest fractions: any deviation from the uniform fractionation increases their number. If this also prolongs the duration of the radiation therapy, undesired consequences, such as cancer cell proliferation, may require delivery of additional dose. This, however, is beyond the scope of this paper.

\section{References}

1. Steel GG. Cell survival as a determinant of tumour response. In: Steel GG, editor. Basic clinical radiobiology. 3rd ed. London: Arnold; 2002. p. 52-63.

2. Joiner MC, Bentzen SM. Time-dose relationships: the linear-quadratic approach. In: Steel GG, editor. Basic clinical radiobiology. 3rd ed. London: Arnold; 2002. p. $120-33$.

3. Horsman MR, Overgaard J. The oxygen effect and tumour microenvironment. In: Steel GG, editor. Basic clinical radiobiology. 3rd ed. London: Arnold; 2002. p. 158-68.

4. Joiner MC. A simple $\alpha / \beta$-independent method to derive fully isoeffective schedules following changes in dose per fraction. Int J Radiat Oncol Biol Phys 2004; 58: 871-5.

5. Bortfeld T, Paganetti H. The biological relevance of daily dose variations in adaptive treatment planning. Int J Radiat Oncol Biol Phys 2006; 64: 899-906. 